CORRECTION

\title{
Antiphospholipid syndrome
}

Karen Schreiber, Savino Sciascia, Philip G. de Groot, Katrien Devreese, Soren Jacobsen,

Guillermo Ruiz-Irastorza, Jane E. Salmon, Yehuda Shoenfeld, Ora Shovman and Beverley J. Hunt

Nature Reviews Disease Primers 4, 17103 (2018)

In the version of the article originally published, Guillermo Ruiz-Irastorza was incorrectly stated as Guillermo Ruiz-Irastroza.

The article has now been corrected. 